\title{
The Future Human: Exploring Posthuman Aesthetics through Digital and Immersive Technologies
}

\author{
Kristina Pulejkova \\ Multimedia Artist \\ 107 Brickfield Studios, Brickfield Road \\ London E3 3LT, UK \\ kristinapulejkova@gmail.com
}

\begin{abstract}
The artistic research explores the post-human aesthetics through the use live action/computer generated animation and Augmented Reality. Drawing on transgenics, biotechnology and bionics, the research proposes two different scenarios of an enhanced human. Effectively, these scenarios are represented through the projects entitled PHOTOPHILIA - a live action/animation video showing a human being that can photosynthesise, and MODIFIED an Augmented Reality experience based on cyborg aesthetics.
\end{abstract}

Posthumanism. Human hybrids. Transgenics. Cyborg aesthetics. Live action/animation hybrid. Augmented Reality.

\section{INTRODUCTION}

Throughout history the fascination with the physical upgrade or enhancement of the human body is evident starting with the myth of Icarus, through Leonardo Da Vinci's machines, the mechanical and organic body explorations of the Bauhaus experimental theatre and the body extensions of contemporary artists Rebecca Horn, ORLAN and Stelarc. Today, such extensions appear in the shape of prosthetics, such as bionic limbs, and electrode implants, installed out of medical necessity. However, with the breakneck pace of technology development, it is not difficult to imagine a future where these alterations will be done without the medical need, but for the desire of enhancing one's abilities. For DeGrazia (2005) "enhancements are interventions to improve human form or function that do not respond to genuine medical needs". This 'enhancement' of the abilities is one of the main traits of the posthuman, i.e. an entity that has been altered and given traits beyond being human.

As Virtual Reality and Augmented Reality become more present and acceptable aspects of our relation with technology, it is vital to ask how the virtual domain is changing our relation to the physical world? To what extend are mixed reality experiences affecting our definition of the body and the self? This research will touch upon these questions and will argue that by accepting different modes of mixed reality, humanity is entering a transition towards post-humanism, towards carbonsilicon symbiosis.

\section{BLURRING THE BOUNDARIES BETWEEN THE NATURAL, HUMAN AND ARTIFICIAL}

It seems that when discussing the posthuman age, the two main strands that are always mentioned are genetic manipulation and enhancement through technological advances, i.e. human-computer interfaces. (Harari 2016, DeGrazia 2005, O’Mahony 2002). Therefore, each project is exploring one of these different scenarios through mixed reality media.

Imagine wearing an implant that has to be medically inserted into your body in order to change your chemical structure on a given time sequence. This is already a common praxis with the widely accepted contraceptive implant whose usage has been on the rise in the UK (NHS Digital 2016), especially with the younger female population. In fact, we are constantly enhancing our performances and choosing to alter the chemical structures of our bodies through use of pharmaceuticals or such implants. However, with the ubiquitous use of VR and AR, one could argue that our perception of where the body ends is also being re-examined. In a way, AR and VR are in fact extending the body through a mixed reality 
environment. As all these enhancements are inherently connected to the body, it was necessary for both projects PHOTOPHILIA and MODIFIED to take the human body as a starting point.

When talking about the body's connection to virtual reality experiences, Hansen (2006) argues that the body assumes the role of an analogue agent in a virtual reality i.e. since the motor reflexes and senses come from the body, in essence every virtual reality experience can be seen as a mixed reality experience. This could be perceived as a way of blurring the boundaries between the body and the technical. Furthermore, by accepting a mixed reality paradigm, one might argue that we are exercising an instance of the extended self.

\section{PHOTOPHILIA - TOWARDS A HUMAN- PLANT ENTITY}

PHOTOPHILIA explores the idea of a future human that had evolved into a human-plant entity able to photosynthesise. The work's title derives from the term 'photophilic', which is used when describing plant life that seeks and/or thrives in sunlight (Oxford Dictionaries 2017).

With the latest developments in biotechnology and transgenics, artists are increasingly exploring ideas of transgenic species as well as hybrid identities and in collaboration with scientists use available technologies to create works that often use biomedia in response to these topics. In 2011, artist Marion Laval-Jeantet conducted a performance piece entitled 'May the Horse Live in Me!' where she injected blood of a horse in her body. The idea behind this bioart project was that the artist wanted to blur the boundaries between herself and the animal, by essentially creating a human-horse hybrid (Miller 2014). By mixing her blood with that of an animal, Laval-Jeantet is also forming a statement on human-animal coexistence.

This coexistence by sharing genetic material across species resonates with my exploration of a fictional human-plant hybrid. In PHOTOPHILIA, the idea is a hybrid human that has evolved within the carbon cycle and has gained the ability to grow photosynthetic cells. By feeding on solar energy, this human-plant prototype has regained the egalitarian status within the biosphere and would enter the age of the Post-Anthropocene. Instead of cultivating nature for its own needs, this human is able to produce oxygen and become a sustainable link in the carbon cycle.

One of the main questions that the piece poses is whether we could look at altering our genome through bioengineering towards mitigating climate change. As humanity is confronted with the threat of Anthropogenic climate change, authors Liao, Sandberg \& Roache (2012) outline various possibilities on human engineering such as making humans red meat intolerant or just shorter in height towards addressing this issue. Furthermore, the authors argue that human engineering should enter a more serious debate as a less risky option to the geo-engineering solutions that are being taken very seriously. In essence, being able to genetically and technologically enhance themselves, humans will be entering a phase of a 'curated' evolution, which for the better or for worse might take some profound changes in the way that humans are experiencing the world.

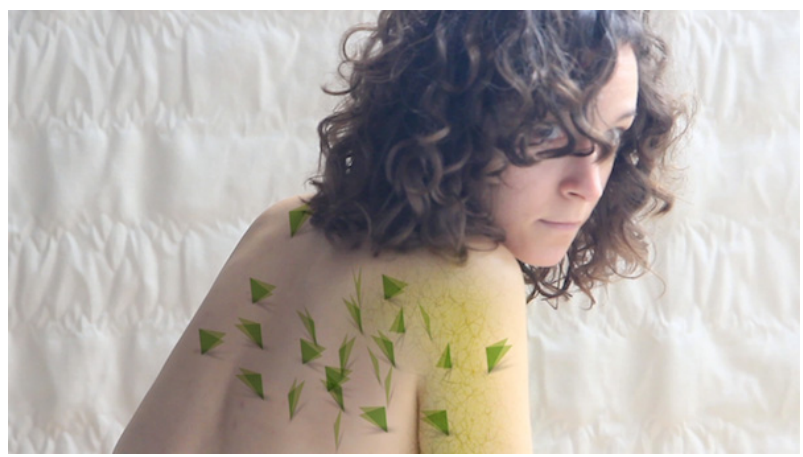

Figure 1: PHOTOPHILIA film still

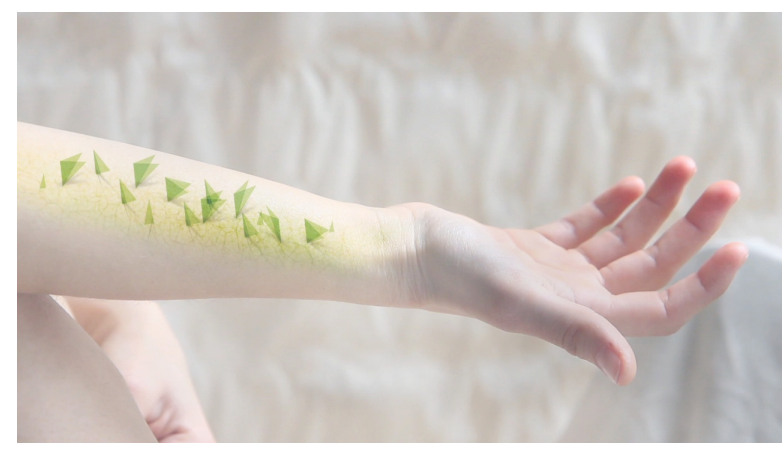

Figure 2: PHOTOPHILIA film still

\subsection{Technical approach}

PHOTOPHILIA is constructed with using a hybrid approach of mixing live action and computer generated animation. Different images of plant cells are stitched into a digital mesh, which is then mapped onto the live action footage of a female model. In the moving image piece, the protagonist is undergoing a transformation from being human to being a human-plant hybrid.

Using motion trackers in Adobe After Effects, the camera and body motion are tracked and then different objects created from the plant cell mesh are mapped onto different parts of the body, further enabling the illusion of emergence of a transgenic species. Alongside the plant cells mesh, numeric data on sea level rise from NASA's Godard Project is also mapped onto the protagonist's eyes, further 
symbolising the ethical thought behind undergoing such transformation.

\subsection{Using live action/animation hybrid towards constructing new realities}

The hybrid mix of live action and computer generated animation used in the piece follows the idea of a mixed reality, in a sense that live action footage serves as the base on which computer generated or virtual objects are mapped in postproduction. As a technique, this hybrid approach has been around since the beginning of animation itself (Crafton 1993). Using the aesthetic behind this technique allows for the virtual or computer generated objects to blend in with reality, further making such a transformation believable, much more so than if the piece were to be fully constructed in an animation software. The footage of an actual human body with all the little imperfections and details of the skin makes the perfect base for the added animation of plant cells to seem realistic, plausible. With the use of this method, the idea was to enable spectators to imagine how that transformation might look like on their own skin, something that might trigger thoughts on more sustainable ways of living. In this respect, I would argue that the hybrid live action/animation technique is the foundation of the much more sophisticated and real-time responsive construct of AR.

\section{MODIFIED - BECOMING A CYBORG}

In MODIFIED, social and psychological aspects of becoming a cyborg are being explored through the use of AR. The piece consists of three different scenarios or modules that are meant to play out onto a participant's skin. Through the use of Augmented Reality, MODIFIED explores the role of the implant in cyborg aesthetics as well as the idea of having the human being a host to the technology. Three different simulations of an implant penetrating into the participant's skin are constructed, each imagining a different aspect of a possible human enhancement. Based on survey research as well as futurist theory, the idea of the piece is to provide different alternatives to what being a cyborg could mean.

Human fascination of becoming more than human or a super-human through enhancing one's body or abilities, as described in literature and mythology, is evident throughout history. This body enhancement has been widely explored in the work of artists like Stelarc and ORLAN. In both artists' practice, the body plays the central role. For Stelarc, "art is a way to explore what it means to be a body" (Miller 2014). For the work 'Ear on Arm', Stelarc in collaboration with scientists grew his own stem cells into an ear mould, which he then implanted under the skin of his arm. The implant itself can be also seen as a transplant as it is his made out of his own cells, but the artist's ultimate idea was to make the output of the ear available online thus extending his body through the web.

Enhancing the body through implants for ORLAN is the equivalent of taking control over her body (Miller 2014). Perhaps the most famous work entitled "The Reincarnation of saint Orlan" is a series of nine operations in which the artist undergoes different face alterations including getting implants on her forehead and cheeks (Featherstone 2000). By altering the body through inserting these implants ORLAN aims to imagine a different body as well as to challenge the Western idea of beauty. Similarly, in MODIFIED I have taken the implant as an outgoing point for reimagining the self and the body.

\subsection{Towards a cyborg future}

By supplying a questionnaire, I was researching my peers' thoughts on posthuman futures and the cyborg in particular. After analysing the answers, the two points made most strongly by the participants were the reluctance to the idea of having an implant and the negative connotation of a cyborg entity. Coined by Manfred E. Clynes and Nathan S. Kline in 1960, the term 'cyborg' is constructed from the word 'cybernetics' and the word 'organism' (O'Mahony 2002). O'Mahony (2002) stresses the crucial role of the second syllable in the term, since the organism, i.e. the human being will be the host of the technology. This idea of having an implant thus rendering the human as host seemed to have 'touched a nerve', making the participants relate the idea of an implant to the notion of the alien or the other.

Usually perceived as a science fiction narrative, the idea of the cyborg in real life is currently entering the debate through the latest development of technologies such as wearables and smart devices. Even though these gadgets are not making us actual cyborgs, they are still enhancing our everyday experiences by adding another layer to our reality. However, there are communities and start-ups like the artist-run Cyborg Foundation and Cyborg Nest - a company that is working on developing new senses through technology, which are working towards blurring the boundaries between carbon and silicon-based entities (Cyborg Foundation 2017, Cyborg Nest 2017).

As previously mentioned, if enhancement is a conscious choice, then one could argue that the choice to enhance one's abilities could be considered a lifestyle. If such a narrative prevails, what are the implications on future socioeconomical structures? Assuming a future scenario 
where human enhancement is seen as commodity, one of the major risks that have been pointed out by researchers is the widening gap between the rich and the poor. By constructing a society of posthumans and the less superior humans there is a risk of dividing the human population in haves and have-nots (Harari 2015, O'Mahony 2002).

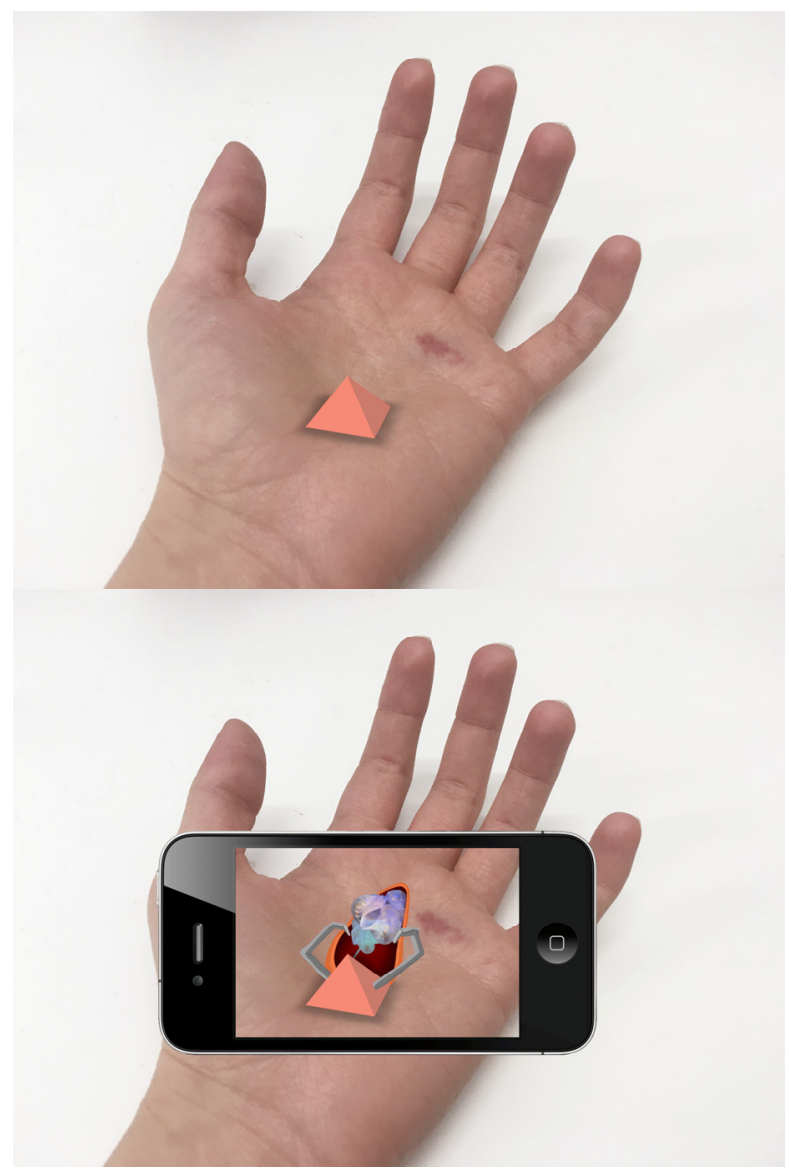

Figure 3: MODIFIED digital sketch of physical module and $A R$ experience

\subsection{Technical approach}

MODIFIED is created with the use of physical objects or modules which are cast out of metal and an Augmented Reality application. Three animations are being produced and each animation is linked to one of the modules. The animations are created in the 3D modelling and animation software Maya and then are mapped onto the physical objects using the game engine Unity. Pointing a smart device's camera onto one of the objects would trigger the corresponding animation.

The interaction process starts with the participant picking up one of the modules and placing it on her or his hand. Being cast in metal, the module has a certain weight that is clearly marking its physical presence on the participant's hand. Furthermore, thanks to the thermal properties of the material, the module's contact with the body will be changing its temperature from cold to warm. This change in temperature would have the potential to enhance the haptic sensation on the participant's skin, further increasing the module's transformation. With the temperature change, the perception of the module will change from being cold and alien, to assuming a similar temperature with its 'host' and therefore feel less alien and more an integrated part of the participant's body.

Once the module is on the participant's hand, with the use of a smartphone or a tablet the corresponding animation will be triggered through the module. The outlining concept behind the animations is to show different ways of how an implant might be inserted into the skin. Through different methods borrowed from medical procedures to science fiction scenarios, the animations are presenting the action of the module being expanded into a device that enables the process of implanting. Even though this process is the piece's main focus, the following animated sequence is actually showing the 'healed' spot on the participant's hand, further implying the successful procedure of becoming a cyborg. A text explanation next to each module explains the purpose of each implant and how this implant might enhance the abilities of its host.

As mentioned earlier in the paper, my research showed that participants are more likely to prescribe a negative connotation to the idea of a cyborg entity. With MODIFIED, the aim is to illustrate the possible benefits of accepting or choosing to enhance one's body through technology. By using humour as well as an aesthetic that is inspired by soft materials like fur, silicone and lights, the piece will essentially invite participants to re-examine their opinions on cyborg aesthetics.

\section{CONCLUSION}

Azuma et al. (2001) define augmented reality systems as:

\begin{abstract}
"An augmented reality (AR) system supplements the real world with virtual (computer-generated) objects that appear to coexist in the same space as the real world [...] we define AR systems as having the following properties: combines real and virtual objects in a real environment, runs interactively, and in real time, and registers (that is, aligns) real and virtual objects with each other".
\end{abstract}

Following this definition, in terms of creating an empathic response or a more realistic simulation, Augmented Reality is a much stronger medium than the live action/animation hybrid video. In PHOTOPHILIA, the viewer was presented with a non-interactive moving image piece that was 
aiming to prompt reflexions on becoming a transgenic entity through the use of the live action/animation hybrid. This hybrid medium enables a sense of a believable reality, but lacks the interactive element of enabling the viewer to experience the transformation in first person.

With the use of Augmented Reality, however, the participant is able to experience the transformation on her or his own skin, viewing the action as it unfolds in real time. Merging the physical and the virtual in real time makes for a much more realistic experience. Furthermore, with the added element of personal experience as well as prompting the haptic senses through the use of a physical object i.e. the module, the notion of empathy and feeling of an extended self is enhanced. By enabling the participants to see the simulation unfold on their own skin through AR, MODIFIED allows the participants to have a more emotional response to the subject of posthuman.

Even though $A R$ is superior to the live action/animation approach in communicating posthuman aesthetics, one could argue that the live action/animation hybrid is still a highly functional medium in creating believable fictional scenarios. The evolution that this hybrid technique had with the constant improvement through its ubiquitous use in the film industry in form of Visual Effects can be seen as an evolution that prompted the mixed reality experience of Augmented Reality. With the use of Augmented Reality humans are experiencing a reality enriched by the virtual, thus enabling them to have a more personal experience on the issue of the posthuman. If $A R$ becomes the norm in which we perceive reality, there will be a need for some form of electronic prosthesis that would enable humans to obtain seamless mixed reality experiences. By doing so, humans wouldn't be leaving the body behind, but enhancing their embodied awareness (Hayles 1999).

\section{REFERENCES}

Azuma, R., Baillot, Y., Behringer, R., Feiner, S., Julier, S., and Maclntyre, B. (2001). Recent advances in augmented reality. IEEE Computer Graphics and Applications, 21(6), pp. 34-47.
Crafton, D. (1993) Before Mickey. The animated film 1898-1928. [Revised edition.] University of Chicago Press, Chicago.

Cyborg Foundation. http://www.cyborgfoundation.com (retrieved 20 March 2017)

Cyborg Nest. https://www.cyborgnest.net (retrieved 20 March 2017)

DeGrazia, D. (2005) Enhancement Technologies and Human Identity. Journal of Medicine and Philosophy, 30, pp. 261-283.

Featherstone, M. (2000) Body Modification. Sage publications, London.

Hansen, M. (2006) Bodies in Code: Interfaces with digital media. Routlege, Taylor \& Francis Group, New York.

Harari, Y. (2015) Homo Deus: A Brief history of Tomorrow. Harvil Secker, London.

Hayles, K. (1999) How we became posthuman: virtual bodies in cybernetics, literature and informatics. University of Chicago Press, Chicago.

Liao, S., Sandberg, A., and Roache, R. (2012) Human Engineering and Climate Change, Ethics, Policy and Environment, 15, pp. 206-221.

Miller, A. (2014) Colliding Worlds: How cuttingedge science is redefining contemporary art. W.W. Norton \& Company, New York.

NHS Digital. (2016) Statistics on Sexual and Reproductive Health Services, England 2015/16. NHS Digital, UK.

O'Mahony, M. (2002) Cyborg: The man-machine. Thames \& Hudson, London.

Oxford Dictionaries (2017). Definition of photophilic in English. English Oxford Living Dictionaries. Oxford University Press, Oxford. https://en.oxforddictionaries.com/definition/photophilic (retrieved 20 March 2017). 